\title{
Qualidade de VidaAssociada ao Tratamento com Radioterapia em Mulheres Acometidas pelo Câncer do Colo do Útero: Revisão Integrativa da Literatura
}

doi: https://doi.org/10.32635/2176-9745.RBC.2021v67n3.1530

\author{
Quality of Life Associated with Radiotherapy Treatment in Women Affected by Cervical Cancer: Integrative Literature Review \\ Calidad de Vida Asociada al Tratamiento con Radioterapia en Mujeres Afectadas por Cáncer de Cuello Uterino: Revisión \\ Integrativa de la Literatura
}

\begin{abstract}
Louyse Jerônimo de Morais ${ }^{\text {; }}$ Aluísio José de Oliveira Monteiro Neto²; João Lucas Pordeus de Menezes³; Maria Gabriela Porfírio Pereira ${ }^{4}$; Yasmin Dantas Pereira ${ }^{5}$; Veriana Márcia da Nóbrega ${ }^{6}$
\end{abstract}

\section{RESUMO}

Introdução: $\mathrm{O}$ câncer do colo do útero $(\mathrm{CCU})$ está entre os cinco tipos de cânceres mais frequentes em mulheres. Inicialmente, o tratamento indicado é a cirurgia. Já, quando a paciente apresenta fatores de risco associados à recidiva local, é feita a radioterapia pélvica adjuvante. Investigar o impacto do tratamento na Qualidade de Vida (QV) das mulheres com CCU pode auxiliar o planejamento de açôes no sentido de reduzir ou evitar danos. Objetivo: Compilar as repercussóes e os fatores que influenciam a QV de mulheres com CCU submetidas ao tratamento radioterápico. Método: Foi realizada uma revisão integrativa da literatura nas bases de dados MEDLINE (PubMed), LILACS e SciELO. Os critérios de inclusão foram artigos dos últimos cinco anos, de acesso aberto, escritos em português, inglês ou espanhol e que abordassem o tema definido total ou parcialmente. Resultados: Foram selecionados 17 artigos, entre os quais predominaram os estudos longitudinais prospectivos $(n=9)$, seguidos dos estudos transversais $(n=5)$ e estudos longitudinais retrospectivos $(\mathrm{n}=3)$. Ademais, houve a utilização de 15 diferentes questionários, sendo mais frequente o European Organization for Research And Treatment of Cancer Quality of Life Questionnaire (EORTC-QLQ-C30), que integrou oito estudos. Conclusáo: A QV das mulheres submetidas à radioterapia foi influenciada por fatores socioeconômicos, educacionais, relaçôes matrimoniais e modalidade da radioterapia. Apesar de os métodos de tratamento terem influenciado fatores individuais, como sintomas físicos e emocionais, não tiveram relação significativa com a QV geral. Estudos devem ser realizados para avaliar os efeitos da radioterapia em longo prazo.

Palavras-chave: Qualidade de Vida; Neoplasias do Colo do Útero/radioterapia; Mulheres.

\section{ABSTRACT}

Introduction: Cervical cancer (CC) is among the five most common cancers in women. Initially, the treatment indicated is surgery. When the patient has risk factors associated with local recurrence, adjuvant pelvic radiotherapy is performed. Investigating the impact of treatment on the quality of life (QoL) of women with CC can help planning actions to reduce or prevent harm. Objective: Compile the repercussions and factors that influence the QoL of women with CC undergoing radiotherapy. Method: A integrative review of the literature was conducted in the MEDLINE (PubMed), LILACS and SciELO databases. The inclusion criteria were articles from the last five years, open access, written in Portuguese, English or Spanish, addressing the theme defined totally or partially. Results: 17 articles were selected, among which prospective longitudinal studies $(n=9)$ predominated, followed by cross-sectional studies $(n=5)$ and retrospective longitudinal studies $(\mathrm{n}=3)$. In addition, 15 different questionnaires were used, the European Organization for Research and Treatment of Cancer Quality of Life Questionnaire (EORTC-QLQ-C30) was more frequent, integrating 8 studies. Conclusion: The QoL of women submitted to radiotherapy was influenced by socioeconomic and educational factors, marital relationships, and the modality of radiotherapy. Although the treatment methods have influenced individual factors, such as physical and emotional symptoms, they had no significant relationship with general QoL. Studies should be conducted to assess the long-term effects of radiotherapy.

Key words: Quality of Life; Uterine Cervical Neoplasms/radiotherapy; Women.

\section{RESUMEN}

Introducción: El cáncer de cuello uterino (CC) se encuentra entre los cinco cánceres más comunes en las mujeres. Inicialmente, el tratamiento indicado es la cirugía. Cuando el paciente presenta factores de riesgo asociados a recidiva local, se realiza radioterapia pélvica adyuvante. Investigar el impacto del tratamiento en la calidad de vida (CV) de las mujeres con CC puede ayudar a planificar acciones para reducir o prevenir daños. Objetivo: Recopilar las repercusiones y factores que influyen en la CV de las mujeres con CC sometidas a radioterapia. Método: Se realizó una revisión integradora de la literatura en las bases de datos MEDLINE (PubMed), LILACS y SciELO. Los criterios de inclusión fueron artículos de los últimos cinco ańos, de acceso abierto, escritos en portugués, inglés o español y que abordaron la temática total o parcialmente definida. Resultados: Se seleccionaron 17 artículos, entre los que predominaron los estudios longitudinales prospectivos $(n=9)$, seguidos de los estudios transversales $(n=5)$ y los estudios longitudinales retrospectivos $(\mathrm{n}=3)$. Además, se utilizaron 15 cuestionarios diferentes, siendo más frecuente el Cuestionario de Calidad de Vida de la Organización Europea para la Investigación y el Tratamiento del Cáncer (EORTC-QLQ-C30), que integró 8 estudios. Conclusión: La CV de las mujeres sometidas a radioterapia estuvo influenciada por factores socioeconómicos, educativos, relaciones maritales y el tipo de radioterapia. Aunque los métodos de tratamiento han influido en factores individuales, como los síntomas físicos y emocionales, no tienen una relación significativa con la CV general. Se deben realizar estudios más amplios para evaluar los efectos a largo plazo de la radioterapia.

Palabras clave: Calidad de Vida; Neoplasias del Cuello Uterino/ radioterapia; Mujeres.

\footnotetext{
1-4,6 Universidade Federal da Paraíba. João Pessoa (PB), Brasil.

${ }^{5}$ Universidade Federal de Campina Grande (PB), Brasil.

'E-mail: louyse.morais@hotmail.com. Orcid iD: https://orcid.org/0000-0003-3499-2776

2E-mail: aluisio_25@hotmail.com. Orcid iD: https://orcid.org/0000-0002-4867-2952

${ }^{3}$ E-mail: pordeus15@gmail.com. Orcid iD: https://orcid.org/0000-0003-4728-8913

4E-mail: mariagabrielaporfirio@gmail.com. Orcid iD: https://orcid.org/0000-0003-2472-2107

5E-mail: yasmindantasp@gmail.com. Orcid iD: https://orcid.org/0000-0001-7713-8022

${ }^{6}$ E-mail: veriana-nobrega@hotmail.com. Orcid iD: https://orcid.org/0000-0003-4494-9229

Endereço para correspondência: Louyse Jerônimo de Morais. Rua Professora Maria da Paz Ferreira Cabral, 149 - Funcionários. João Pessoa (PB), Brasil. CEP 58079-140. E-mail: louyse.morais@hotmail.com
} 


\section{INTRODUÇÃO}

O câncer do colo do útero (CCU) está entre os cinco tipos de cânceres mais frequentes em mulheres, sendo estimado que, somente no ano de 2018, ocorreram cerca de 18 milhôes de novos casos e 9,6 milhóes de mortes ${ }^{1}$. Por outro lado, há exames de fácil acesso, como o Papanicolau, com caráter preventivo, que possibilitam diagnóstico precoce e maiores chances de cura à paciente ${ }^{2}$.

A infecção pelo Papilomavírus Humano (HPV) constitui a principal causa para o desenvolvimento do câncer cervical. Os 15 tipos de HPV que apresentam um forte potencial oncogênico incluem HPV 16, 18, 31, 33, $35,39,45,51,52,56,58,59,68,73$ e 82 . Esses tipos são considerados de alto risco e representam a etiologia de 95\% de todos os cânceres de CCU. Os tipos 16 e 18, especificamente, são responsáveis por cerca de $90 \%$ de todos os $\operatorname{casos}^{3,4}$.

Fatores sociais e econômicos são de grande relevância, visto que, em alguns países com baixo e médio Índice de Desenvolvimento Humano (IDH), o CCU é um dos mais incidentes. No que se refere ao Brasil, essa neoplasia é a mais incidente na Regiāo Norte e a segunda mais incidente nas Regiốes Nordeste e Centro-Oeste. Já nas Regiôes Sul e Sudeste, ela ocupa, respectivamente, a quarta e a quinta posição ${ }^{5,1}$.

O diagnóstico do câncer poderá anteceder ao emprego de determinadas modalidades terapêuticas, como a cirurgia, radioterapia, quimioterapia e hormonioterapia, sendo iniciada uma etapa por vezes desafiadora na vida das mulheres portadoras do $\mathrm{CCU}^{6}$. Para orientar essa nova fase, a Organização Mundial da Saúde (OMS) divide o tratamento do câncer em dois tipos: sistêmico - hormonioterapia e quimioterapia - e locorregional - cirurgia e radioterapia. A conduta terapêutica será guiada pelo tipo do câncer, estadiamentos clínico e patológico, disponibilidade de infraestrutura adequada e de profissionais especializados, assim como fatores individuais, como a idade e desejo de ter filhos ${ }^{7,8}$.

Para o CCU, em fases iniciais, o tratamento indicado é a cirurgia. Já, quando o paciente apresenta fatores de risco associados à recidiva local, é feita a radioterapia pélvica adjuvante. $\mathrm{Na}$ presença de comprometimento linfonodal, parametrial ou das margens cirúrgicas, faz-se quimioterapia associada à radioterapia. Quando o câncer é localmente avançado ou em tumores muito volumosos, recomenda-se a associação de quimioterapia com radioterapia 9 .

Pacientes submetidos à radioterapia pélvica podem apresentar dano tecidual a ponto de haver oclusão de vasos sanguíneos, trombose e neovascularização, bem como atrofia e contração do tecido em virtude do aumento da proliferaçáo de fibroblastos. Adicionalmente, podem ser descritas como principais complicaçôes da radioterapia no trato urinário a ureterite actínica, fístula vesicovaginal, cistite actínica e hematúria, assim como complicaçóes no intestino e no reto, sendo neste a mais frequente a retocolite ulcerativa ${ }^{10,11}$.

Apesar das várias consequências negativas supracitadas, poucos estudos enfatizam o impacto na Qualidade de Vida (QV) das mulheres sujeitas ao tratamento de câncer cervical. O presente estudo busca abordar os efeitos secundários da radioterapia na $\mathrm{QV}$, cuja definição, de acordo com a OMS, são "as percepçóes dos indivíduos de sua posição na vida no contexto da cultura e sistemas de valores em que vivem e em relação aos seus objetivos, expectativas, padróes e preocupaçóes" ${ }^{12}$. Investigar o impacto do tratamento na QV das mulheres com CCU pode auxiliar o planejamento de açóes no sentido de reduzir ou evitar danos.

Sendo assim, a pesquisa objetivou, por meio de uma revisão bibliográfica da literatura, compilar as repercussóes e os fatores que influenciam a $\mathrm{QV}$ de mulheres com CCU submetidas ao tratamento radioterápico. Para avaliar tais repercussóes, foram utilizados questionários específicos, a fim de mensurar a QV, e os parâmetros incluíram: QV sexual, bem-estar físico e psicológico, além de prejuízos de natureza orgânica, social e profissional.

\section{MÉTODO}

Trata-se de uma revisão integrativa da literatura, a qual propóe responder à seguinte pergunta de pesquisa: "Quais são as repercussôes e os fatores que podem influenciar na QV de mulheres de todas as faixas etárias, acometidas pelo CCU, que se submetem ao tratamento com radioterapia?". O processo realizado foi desenvolvido em etapas, tais como: escolha das bases de dados, definição dos descritores, estabelecimento dos critérios de inclusão e exclusão, coleta de dados, leitura dos títulos, palavras-chave e resumos e, após nova etapa de exclusāo, leitura dos artigos completos. Esta última etapa também permitiu a exclusão de artigos cujos conteúdos não foram pertinentes ao trabalho.

As bases de dados escolhidas foram: MEDLINE (PubMed), LILACS e SciELO. Os descritores foram definidos a partir dos Descritores em Ciências da Saúde (DeCS) e Medical Subjects Headings (MeSH), e consistiram em Uterine Cervical Neoplasms, Radiotherapy e Quality of life. Os três foram unidos pelo operador booleano AND em todas as bases de dados.

Os critérios de inclusão estabelecidos foram: (i) artigos publicados nos últimos cinco anos (2016 a 2020); (ii) de acesso aberto; (iii) escritos nos idiomas português, inglês 
ou espanhol; e (iv) que abordassem total ou parcialmente o tema definido. Foram excluídos da pesquisa artigos de revisôes de literatura, revisões integrativas e sistemáticas, assim como aqueles em que o desfecho primário não fosse diretamente relacionado ao tema da pesquisa.

\section{RESULTADOS}

Após a aplicação dos critérios de inclusão e exclusão preestabelecidos, bem como exclusão dos artigos duplicados e dos que náo condiziam com o objetivo proposto da revisão, restaram 17 estudos. O fluxograma desse processo está ilustrado na Figura 1.

Os artigos selecionados foram avaliados de acordo com a abordagem de repercussão na QV utilizada por cada um deles - função sexual, emocional, física, condiçóes socioeconômicas e QV geral. Alguns estudos utilizaram questionários voltados apenas para uma função, como o Female Sexual Function Index (FSFI), que avalia apenas a função sexual, mas outros utilizaram questionários bem consolidados, que avaliam diferentes aspectos da QV, como o European Organization for Research And Treatment of Cancer Quality of Life Questionnaire (EORTC-QLQ-C30).

Desse modo, foram selecionados 12 estudos longitudinais, sendo três retrospectivos e nove prospectivos, além de cinco estudos transversais. A Tabela 1 apresenta os dados e as principais conclusôes dos estudos incluídos na revisão.

\section{DISCUSSÃO}

A avaliação da QV por mulheres submetidas à radioterapia para tratamento de CCU é influenciada por inúmeros fatores, que podem estar relacionados direta e/ou indiretamente ao tratamento. Entre tais agentes modificadores da percepção da paciente em relação à $\mathrm{QV}$, foram destacados nesta revisão: idade, escolaridade, hábitos, profissão, condição socioeconômica, status civil, estadiamento do câncer e tipo de tratamento adotado.

Em relação à idade e ao grau de escolaridade, a pesquisa de Kyei et al. ${ }^{13}$ formulou a hipótese de que as pacientes mais jovens e instruídas possuíam maior atenção e preocupação sobre os efeitos colaterais do tratamento radioterápico, concluindo que o bem-estar físico de mulheres com CCU foi afetado pela idade e nível de escolaridade. Essa constatação foi corroborada, ainda, por Singh et al. ${ }^{14}$, cujo estudo identifica o nível educacional, junto ao uso de tabaco e menor grau de diferenciação do tumor, como um fator independente que afeta negativamente a $\mathrm{QV}$ geral.

Quanto às consequências da realização de atividade profissional entre as pacientes em tratamento radioterápico, o estudo transversal conduzido por Zhou et al. ${ }^{15}$ identificou uma correlação entre ocupação profissional e atividade sexual, nunca antes relatada na literatura: sobreviventes de câncer cervical que trabalhavam em funçōes administrativas, burocráticas ou de gerenciamento apresentaram melhor função sexual do que o restante das pacientes que ocupavam outras funçóes laborais, o que pode estar relacionado ao nível de escolaridade e a menores níveis de exposição à radioterapia.

A pesquisa de Correia et al. ${ }^{16}$ utilizou o questionário abreviado The World Health Organization Quality of Life Assessment (WHOQOL-bref) para medir a QV em mulheres que realizaram cirurgia, quimioterapia ou radioterapia. Foi mostrada uma relação negativa entre as piores condiçóes socioeconômicas, falta de oportunidade

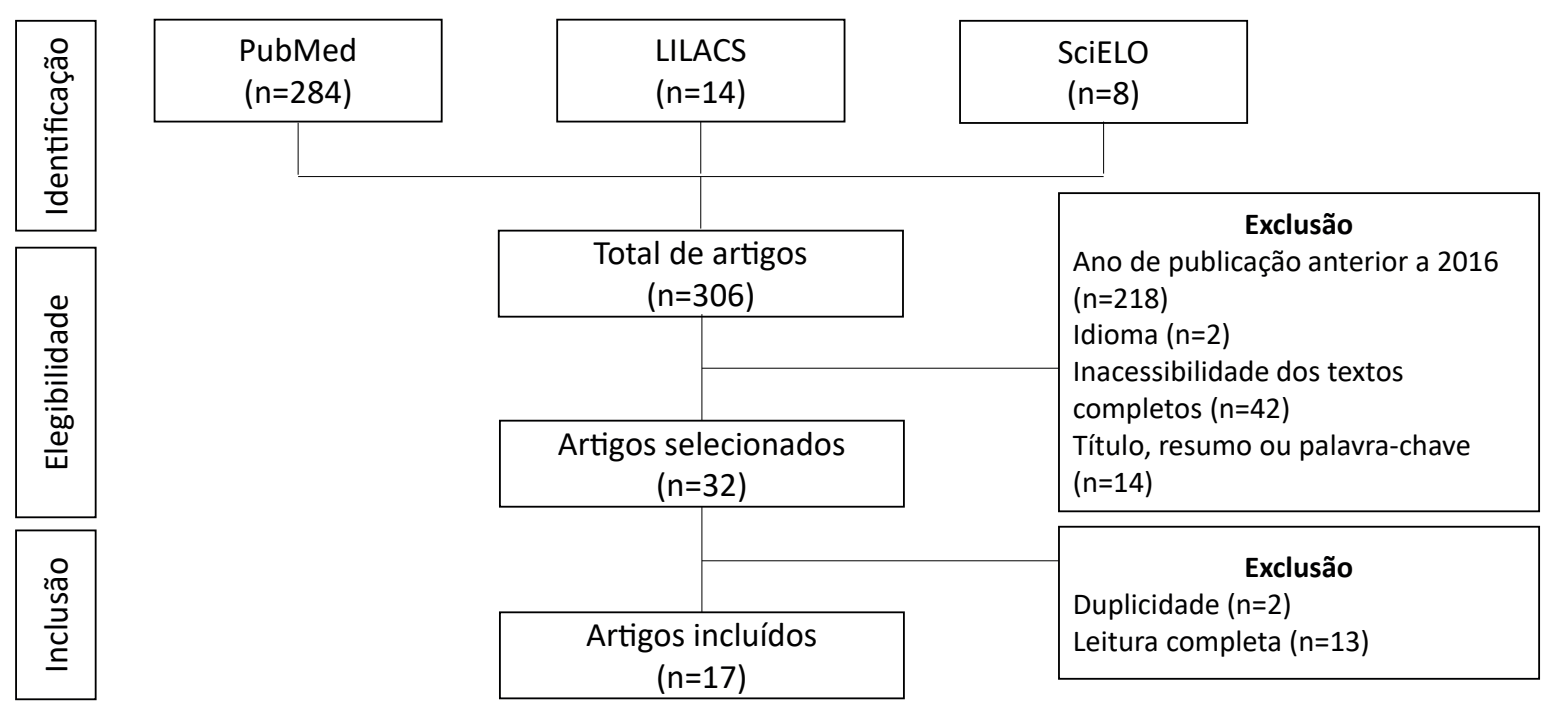

Figura 1. Fluxograma do processo de seleção dos artigos 
Tabela 1. Características dos estudos selecionados para a revisão

\begin{tabular}{|c|c|c|c|c|c|}
\hline Autor/ano & Delineamento & País & $\mathbf{N}$ & Questionários & Conclusões \\
\hline $\begin{array}{l}\text { Akbaba et } \\
\text { al., } 2018\end{array}$ & $\begin{array}{l}\text { Longitudinal } \\
\text { retrospectivo }\end{array}$ & Alemanha & 83 & $\begin{array}{l}\text { EORTC } \\
\text { QLQ-C30 } \\
\text { e EORTC } \\
\text { QLQ-CX24 }\end{array}$ & $\begin{array}{l}\text { O grau de efeitos colaterais agudos da } \\
\text { radioquimioterapia se correlacionou } \\
\text { com a extensão de sintomas crônicos. O } \\
\text { funcionamento sexual/vaginal foi pior em } \\
\text { pacientes com tumores maiores }\end{array}$ \\
\hline $\begin{array}{l}\text { Castaneda } \\
\text { et al., } 2019\end{array}$ & Transversal & Brasil & 116 & FACT-Cx & $\begin{array}{l}\text { A maior prevalência de incapacidade } \\
\text { para as mulheres com CCU se deu no } \\
\text { sistema reprodutivo. Em relação à QV, foi } \\
\text { observada uma deterioração relacionada } \\
\text { a temores em relação à vida sexual e } \\
\text { função urinária }\end{array}$ \\
\hline $\begin{array}{l}\text { Cerentini et } \\
\text { al., } 2019\end{array}$ & $\begin{array}{l}\text { Longitudinal } \\
\text { prospectivo }\end{array}$ & Brasil & 88 & $\begin{array}{l}\text { EORTC } \\
\text { QLQ-C30 }\end{array}$ & $\begin{array}{l}\text { Não houve diferença entre os grupos } \\
\text { intervenção (dilatador vaginal) e controle } \\
\text { em relação à QV. Os prejuízos na QV } \\
\text { começaram a melhorar } 3-6 \text { meses após o } \\
\text { final da RT }\end{array}$ \\
\hline $\begin{array}{l}\text { Correia et } \\
\text { al., } 2020\end{array}$ & Transversal & Brasil & 46 & FSFI & $\begin{array}{l}\text { Mulheres submetidas à RT apresentaram } \\
\text { mais sintomas sexuais comparadas } \\
\text { àquelas tratadas com cirurgia e QT }\end{array}$ \\
\hline $\begin{array}{l}\text { Correia et } \\
\text { al., } 2018\end{array}$ & $\begin{array}{l}\text { Longitudinal } \\
\text { prospectivo }\end{array}$ & Brasil & 46 & WHOQOL-100 & $\begin{array}{l}\text { A QV esteve associada às condições } \\
\text { clínicas, socioeconômicas e de saúde, } \\
\text { estando ligadas ao tipo de tratamento que } \\
\text { realizaram (o diagnóstico tardio refletiu-se } \\
\text { no tratamento com RT e QT) }\end{array}$ \\
\hline $\begin{array}{l}\text { Klopp et } \\
\text { al., } 2018\end{array}$ & $\begin{array}{l}\text { Longitudinal } \\
\text { prospectivo }\end{array}$ & $\begin{array}{l}\text { Estados } \\
\text { Unidos }\end{array}$ & 278 & $\begin{array}{l}\text { EPIC; } \\
\text { PRO-CTCAE; } \\
\text { FACT-Cx e } \\
\text { EQ-5D }\end{array}$ & $\begin{array}{l}\text { A IMRT resultou em um impacto menor } \\
\text { na função intestinal e na função urinária. } \\
\text { Questionários de QV demonstraram } \\
\text { que pacientes tratadas com IMRT } \\
\text { também tiveram menor diminuição do } \\
\text { funcionamento físico }\end{array}$ \\
\hline $\begin{array}{l}\text { Kwak et al., } \\
2017\end{array}$ & $\begin{array}{l}\text { Longitudinal } \\
\text { retrospectivo }\end{array}$ & Coreia & 136 & LENT/SOMA & $\begin{array}{l}\text { Os resultados mostraram superioridade da } \\
\text { IMRT sobre a RT. Com toxicidade reduzida, } \\
\text { a IMRT aumentou a QV dos pacientes e } \\
\text { a adesão ao tratamento. Os resultados } \\
\text { sugerem que, com IMRT, o escalonamento } \\
\text { seletivo da dose em RT para a pelve será } \\
\text { praticável e dará a chance de melhores } \\
\text { resultados clínicos }\end{array}$ \\
\hline $\begin{array}{l}\text { Kyei et al., } \\
2020\end{array}$ & Transversal & Gana & 120 & FACT-G & $\begin{array}{l}\text { Pacientes com câncer em estágio inicial } \\
\text { relataram escores de QV geral mais altos } \\
\text { do que aqueles com apresentação tardia } \\
\text { da doença }\end{array}$ \\
\hline $\begin{array}{l}\text { Li et al., } \\
2017\end{array}$ & $\begin{array}{l}\text { Longitudinal } \\
\text { prospectivo }\end{array}$ & China & 480 & $\begin{array}{l}\text { DT; HHI; } \\
\text { FACT-Cx e JCS }\end{array}$ & $\begin{array}{l}\text { Fornecer orientação sobre o nível de } \\
\text { esperança e estilo de enfrentamento pode } \\
\text { ajudar os pacientes a compreender a } \\
\text { doença, reduzir o sofrimento psicológico } \\
\text { e melhorar o nível de esperança para } \\
\text { enfrentar a doença de maneira positiva }\end{array}$ \\
\hline $\begin{array}{l}\text { Mohanty et } \\
\text { al., } 2018\end{array}$ & $\begin{array}{l}\text { Longitudinal } \\
\text { prospectivo }\end{array}$ & Índia & 64 & $\begin{array}{l}\text { EORTC } \\
\text { QLQ-C30 } \\
\text { e EORTC } \\
\text { QLQ-CX24 }\end{array}$ & $\begin{array}{l}\text { A IMRT adjuvante pode estar associada } \\
\text { à melhora da QV em longo prazo. A } \\
\text { magnitude do prejuízo para a maioria dos } \\
\text { domínios de QV é menor, e o uso de IMRT } \\
\text { resulta em pontuações de QV clinicamente } \\
\text { melhores em comparação com a 3D-CRT }\end{array}$ \\
\hline
\end{tabular}


Tabela 1. continuação

\begin{tabular}{|c|c|c|c|c|c|}
\hline Autor/ano & Delineamento & País & $\mathbf{N}$ & Questionários & Conclusões \\
\hline $\begin{array}{l}\text { Sabulei } \\
\text { e Maree, } \\
2019\end{array}$ & Transversal & $\begin{array}{l}\text { África do } \\
\text { Sul }\end{array}$ & 153 & $\begin{array}{l}\text { EORTC } \\
\text { QLQ-C30 e } \\
\text { EORTC QLQ- } \\
\text { CX24 }\end{array}$ & $\begin{array}{l}\text { O CCU e seu tratamento tiveram } \\
\text { influência negativa na QV e } \\
\text { influenciaram todos os domínios da } \\
\text { vida. O funcionamento social foi o mais } \\
\text { afetado; dificuldades financeiras foram } \\
\text { problemáticas, especialmente durante o } \\
\text { período de tratamento; frequência urinária } \\
\text { e insônia permaneceram problemáticas; e } \\
\text { o inchaço dos pés aumentou com o tempo }\end{array}$ \\
\hline $\begin{array}{l}\text { Shankar et } \\
\text { al., } 2020\end{array}$ & $\begin{array}{l}\text { Longitudinal } \\
\text { prospectivo }\end{array}$ & Índia & 85 & LENT/SOMA & $\begin{array}{l}\text { O público mais afetado pela RT em } \\
\text { concomitância com a QT foi o jovem. O } \\
\text { estágio avançado do câncer e o tempo } \\
\text { de acompanhamento foram diretamente } \\
\text { proporcionais à morbidade relacionada à } \\
\text { função sexual }\end{array}$ \\
\hline $\begin{array}{l}\text { Silveira et } \\
\text { al., } 2016\end{array}$ & $\begin{array}{l}\text { Longitudinal } \\
\text { prospectivo }\end{array}$ & Brasil & 16 & $\begin{array}{l}\text { EORTC } \\
\text { QLQ-C30 }\end{array}$ & $\begin{array}{l}\text { A função emocional foi o item mais } \\
\text { afetado, sugerindo que o diagnóstico, } \\
\text { tratamento e efeitos colaterais impactam } \\
\text { de forma importante o emocional }\end{array}$ \\
\hline $\begin{array}{l}\text { Singh et } \\
\text { al., } 2019\end{array}$ & $\begin{array}{l}\text { Longitudinal } \\
\text { prospectivo }\end{array}$ & Índia & 90 & $\begin{array}{l}\text { EORTC } \\
\text { QLQ-C30 e } \\
\text { EORTC QLQ- } \\
\text { CX24 }\end{array}$ & $\begin{array}{l}\text { Educação, uso de tabaco, grau de } \\
\text { diferenciação do tumor, tamanho do } \\
\text { tumor e estágio do câncer foram as } \\
\text { variáveis independentes que mais } \\
\text { afetaram a QV }\end{array}$ \\
\hline $\begin{array}{l}\text { Yang et al., } \\
2020\end{array}$ & $\begin{array}{l}\text { Longitudinal } \\
\text { retrospectivo }\end{array}$ & China & 97 & $\begin{array}{l}\text { FSFI e EORTC } \\
\text { QLQ-C30 }\end{array}$ & $\begin{array}{l}\text { Diferentes terapias adjuvantes pós- } \\
\text {-operatórias causaram diferenças } \\
\text { significativas na QV em longo prazo. } \\
\text { Dificuldades financeiras impactaram a QV } \\
\text { de pacientes jovens com câncer pós-RT } \\
\text { adjuvante. Pacientes jovens que fizeram } \\
\text { RT adjuvante tiveram pior QV global após } \\
5 \text { anos }\end{array}$ \\
\hline $\begin{array}{l}\text { Yavas et } \\
\text { al., } 2017\end{array}$ & $\begin{array}{l}\text { Longitudinal } \\
\text { prospectivo }\end{array}$ & Turquia & 100 & $\begin{array}{l}\text { EORTC } \\
\text { QLQ-C30; } \\
\text { EORTC QLQ- } \\
\text { CX24 e HADS }\end{array}$ & $\begin{array}{l}\text { Não houve efeito adverso importante na } \\
\text { QVRS de longo prazo resultantes da RT. A } \\
\text { piora aguda da QVRS durante a fase de } \\
\text { tratamento melhorou em longo prazo }\end{array}$ \\
\hline $\begin{array}{l}\text { Zhou et al., } \\
2016\end{array}$ & Transversal & China & 140 & $\begin{array}{l}\text { FACT-Cx; } \\
\text { FACIT-Sp e FSFI }\end{array}$ & $\begin{array}{l}\text { A QV de sobreviventes de CCU foi } \\
\text { considerada baixa. Houve também } \\
\text { prejuízo com relação às funções sexuais e } \\
\text { outras complicações relacionadas à QV }\end{array}$ \\
\hline
\end{tabular}

Legendas: FSFI = Female Sexual Function Index; EORTC QLQ-C30 e EORTC QLQ-CX24 = European Organization for Researchand Treatment of Cancer Quality of Life Questionnaire; FACT-Cx e FACT-G = Functional Assessment of Cancer Therapy; FACIT-Sp = Functional Assessment of Chronic Illness Therapy-Spiritual Wellbeing, EPIC $=$ Expanded Prostate Cancer Index Composite; $\mathrm{PRO}-\mathrm{CTCAE}=$ Patient-Reported Outcomes-Common Terminology Criteria for Adverse Events; DT $=$ Distress thermometer $; \mathrm{HHI}=$ Herth Hope Index; JCS = Coping Style Scale; HADS = Hospital Anxiety and Depression Scale; WHOQOL-100 = Instrument for the Assessment of the Quality of Life of the World Health Organization; LENT/SOMA = Escala de Late Effects of Normal Tissue/Subjective-Objective-Management-Analytic; 3D-CRT $=$ Radioterapia conformacional tridimensional; IMRT $=$ Radioterapia de intensidade modulada; EQ-5D = EuroQol 5 Dimensions; RT = Radioterapia; $\mathrm{QT}=$ Quimioterapia; QV = Qualidade de Vida; QVRS = Qualidade de Vida Relacionada à Saúde; CCU = Câncer do colo do útero.

de lazer, presença de comorbidades e realização de tipos de terapia que incluem radioterapia. Nesse trabalho, os piores resultados físicos, funcionais e sociais foram correlacionados com a radioterapia.

Além disso, foi mostrado, ainda, que as dificuldades financeiras, pelos gastos com a radioterapia e outros fatores, influenciaram significativamente a $\mathrm{QV}$ das mulheres submetidas à radioterapia adjuvante, isto ocorreu também em outros estudos ${ }^{15,17,18}$. Portanto, pacientes jovens que fizeram radioterapia adjuvante tiveram pior $\mathrm{QV}$ global em longo prazo após cinco anos. Isso, parcialmente, vai de encontro com o estudo de Mohanty et al. ${ }^{19}$, que revelou redução significativa nos escores de QV após irradiação pélvica em um primeiro momento, mostrando melhora gradual e definitiva com o tempo, a depender da modalidade de radioterapia. 
De acordo, ainda, com Kyei et al. ${ }^{13}$, as mulheres casadas pontuaram mais alto em termos de pontuaçáo média do que as mulheres solteiras; ou seja, a presença do companheiro teve um papel no bem-estar emocional das pacientes. Esse dado também foi obtido em outros estudos, que indicaram que esse apoio é um fator importante para o enfrentamento e tratamento das doenças, bem como para a imagem corporal e atividade sexual ${ }^{19-21}$. Em pacientes tratadas por meio de cirurgia combinada à radioterapia, houve maior prejuízo ao domínio emocional, em comparação com aquelas submetidas a outras opçóes terapêuticas - apenas radioterapia; radioterapia e quimioterapia; radioterapia, quimioterapia e cirurgia.

Em concomitância com os resultados dos estudos supracitados, Li et al. ${ }^{20}$ apontaram, em estudo com 480 pacientes, que pacientes com câncer cervical, mesmo apresentando alta morbidade de sofrimento psicológico (68\%), apresentaram níveis médios e altos de esperança de cura durante a radioterapia. Maior idade, maior renda familiar, seguro médico completo, relaçóes matrimoniais e menor nível de dor durante o tratamento também indicaram maiores níveis de esperança e, por conseguinte, melhor QV, na amostra estudada.

Os resultados do ensaio clínico conduzido por Sabulei e $\mathrm{Maree}^{18}$ mostraram que o tratamento radioterápico, em associação ou não com a quimioterapia, influenciou negativamente todos os domínios de QV das pacientes. O domínio social foi o mais afetado, não apresentando melhora significativa ao longo do tempo.

Em relação à QV geral das pacientes, quanto mais inicial o estágio do câncer, maior o escore de $\mathrm{QV}^{13}$. Outros estudos também mostraram essa relaçáa ${ }^{14,20}$. Além disso, a função sexual e vaginal foi estatisticamente significativamente pior em tumores mais avançados ( $\mathrm{T} 3 \mathrm{e}$ T4) em comparação aos tumores mais iniciais (T1 e T2 $)^{22}$.

Quanto às modalidades de tratamento da radioterapia, Klopp et al. ${ }^{23}$ observaram em seu estudo que a radioterapia de intensidade modulada (IMRT) resultou em menos impacto na função intestinal e urinária durante o tratamento do que a radioterapia padrão. Ainda nesse contexto, as métricas de QV demonstraram que os pacientes tratados com IMRT também tiveram um declínio menor na função física e preocupaçôes adicionais relacionadas ao tratamento durante o curso da radioterapia.

Em consonância com o estudo anterior, Mohanty et al. ${ }^{19}$ inferiram que a IMRT pode estar associada a melhores escores de QV em longo prazo. A magnitude do prejuízo para a maioria dos domínios da QV foi menor e o uso de IMRT resultou em pontuaçôes de QV clinicamente e estatisticamente significativas, em comparaçáo com a radioterapia conformada tridimensional adjuvante (3DCRT), outra modalidade de radioterapia.
Em um estudo prospectivo longitudinal ${ }^{24}$, realizado com cinco mulheres irradiadas na pelve, a QV das pacientes foi considerada boa. $\mathrm{O}$ escore obtido em relação à função emocional das mulheres foi o mais negativo do estudo, evidenciando-se que a soma de diversos fatores, tais como o diagnóstico, o tratamento radioterápico e os efeitos colaterais, impacta de forma significativa a saúde mental das mulheres. Além disso, mostrou-se que a associação concomitante da quimioterapia ao tratamento também afeta a função física das pacientes. $\mathrm{O}$ estudo de coorte de Akbaba et al. ${ }^{22}$ mostrou que a radioquimioterapia primária reduz a $\mathrm{QV}$ em longo prazo, embora seja um tratamento eficaz.

Entre os efeitos adversos infligidos pelo tratamento radioterápico às pacientes acometidas por $\mathrm{CCU}$, destacam-se prejuízos à saúde sexual. Dessa forma, a função sexual das mulheres pós-tratamento para CCU foi avaliada por alguns autores por meio do FSFI. Foi encontrada uma maior prevalência de sintomas sexuais em mulheres submetidas à radioterapia em comparação com aquelas tratadas com cirurgia e quimioterapia ou apenas cirurgia sem adjuvância radioterápica. Entre os sintomas, destacou-se frigidez, falta de lubrificação, de excitação, de orgasmo, de libido e dispareunia ${ }^{13,18}$. Verificou-se que se a mulher não recebesse radioterapia pós-cirúrgica, as disfunçôes sexuais poderiam ser revertidas, desde que não se tivesse realizado salpingooforectomia bilateral no procedimento cirúrgico.

Ainda com base nos escores FSFI, Zhou et al. ${ }^{15}$ identificaram que $70 \%$ das pacientes do seu estudo apresentaram disfunção sexual após o tratamento oncológico (radioterapia, quimioterapia e histerectomia). Questóes psicológicas e cognitivas se mostraram como fortes modificadores na experiência e na QV sexual das pacientes, sobretudo preocupaçôes quanto à recorrência do câncer $(63,8 \%)$ e ao medo de dor e sangramento em virtude do sexo $(31,9 \%)$. Tais resultados também estiveram presentes no estudo de Correia et al..$^{25}$, o que se tornou um motivo para que as mulheres evitassem o sexo após o tratamento contra CCU.

Adicionalmente, o estudo realizado por Castaneda et al. ${ }^{26}$ constatou que incapacidades relacionadas a estruturas do sistema reprodutivo apresentaram maior prevalência entre as mulheres, seguidas de problemas nas funçóes de energia e do impulso. Além disso, pareceu haver especialmente temores relacionados à vida sexual e a problemas ligados à função urinária. Cabe ressaltar que, nesse estudo, a radioterapia não foi utilizada de forma isolada, e sim combinada com a quimioterapia.

Nessa pesquisa, uma das incapacidades relacionadas aos órgãos do trato genital feminino foi a sensação de estreitamento e encurtamento vaginal. Além disso, a 
estenose vaginal após o tratamento também é uma queixa comum $^{26}$. Nesse sentido, Cerentini et al. ${ }^{27}$ buscaram avaliar o benefício de dilatadores vaginais durante o tratamento radioterápico no canal vaginal em virtude de câncer cervical. No entanto, não houve diferença na QV das mulheres que usaram os dilatadores em comparação ao grupo controle.

Um estudo prospectivo, conduzido por Shankar et al. ${ }^{28}$, observou que a combinação da radioterapia com a cirurgia elevou a disfunção e insatisfação sexuais, principalmente em mulheres mais jovens. Notou-se que as queixas mais comuns, em ordem decrescente, foram a dispareunia, piora na qualidade da vida sexual e diminuição do interesse pelo sexo. Assim, o estudo observou que, no futuro, a ênfase sobre o dever de informar sobre o risco de alteraçóes sexuais e gerais após a radioterapia deverá ser reforçada e há necessidade de que se construam escalas mais abrangentes abordando as perturbaçóes físicas e psicológicas ${ }^{28}$.

Uma vez caracterizados os efeitos adversos do tratamento radioterápico, bem como sua a repercussão na forma como as pacientes avaliam a QV durante o tratamento, é possível examinar as mudanças na avaliação da QV pelas pacientes no período posterior ao tratamento radioterápico. No ensaio clínico de Yavas et al. ${ }^{29}$, foi analisada a QV de pacientes com câncer ginecológico imediatamente antes da radioterapia, pós-radioterapia e durante 24 meses de acompanhamento pós-radioterapia. Em suma, concluiu-se que nenhum efeito adverso significativo em longo prazo na QV geral dos pacientes resultou da radioterapia. Além disso, percebeu-se que os escores de atividade sexual e o prazer sexual deterioraram-se no período pós-radioterapia; no entanto, no $24^{\circ}$ mês de acompanhamento, ambos os escores melhoraram significativamente.

O estudo feito por Singh et al. ${ }^{14}$ observou melhora dos escores relacionados à escala funcional de QV geral após seis meses de tratamento radioterápico, associado ou não à quimioterapia, em comparação com o momento anterior ao início do tratamento (linha de base). Sobressaíram-se as funções física e emocional. Todavia, foi considerado que tais melhorias ocorreram sobretudo em razão da diminuição da preocupação com o diagnóstico de câncer. Por outro lado, foi constatada diminuição do prazer sexual e presença de disfunção sexual significativas. Esses dados corroboram o estudo de Yavas et al. ${ }^{29}$, que mostrou redução dos níveis de ansiedade e depressão das pacientes ao longo do tempo. Esses autores ${ }^{17}$, no entanto, verificaram que as funçōes físicas e emocionais de mulheres submetidas à radioterapia adjuvante não melhoraram com o passar do tempo.

\section{CONCLUSÃO}

O tratamento do CCU realizado com radioterapia está associado a uma maior prevalência de disfunçóes sexuais, especialmente em mulheres mais jovens. A combinação de radioterapia com quimioterapia ocasionou mais sintomas físicos; já a combinação com a cirurgia, maior impacto emocional. Apesar disso, as formas de tratamento náo apresentaram relação estatisticamente significativa com a QV geral.

O impacto positivo na QV foi observado em mulheres com maior renda familiar, maiores níveis educacionais, relaçôes matrimoniais e menor nível de dor durante o tratamento. Em contrapartida, os fatores relacionados a piores condiçôes socioeconômicas, falta de oportunidade de lazer, comorbidades e estado civil solteira impactaram negativamente na QV das mulheres.

Em longo prazo, houve contradição entre os estudos quanto à melhoria das funções física e emocional. Dessa forma, sugere-se que mais pesquisas sejam realizadas, a fim de entender melhor os efeitos da radioterapia nesse aspecto ao longo do tempo.

\section{CONTRIBUIÇÕES}

Louyse Jerônimo de Morais, João Lucas Pordeus de Menezes, Aluísio José de Oliveira Monteiro Neto, Maria Gabriela Porfírio Pereira e Yasmin Dantas Pereira contribuíram na concepção e/ou no planejamento do estudo; na obtenção, análise e interpretação dos dados; assim como na redação e revisão crítica. Veriana Márcia da Nóbrega contribuiu na redação e revisão crítica. Todos os autores aprovaram a versão final a ser publicada.

\section{DECLARAÇÃO DE CONFLITO DE INTERESSES}

Nada a declarar.

\section{FONTES DE FINANCIAMENTO}

Não há.

\section{REFERÊNCIAS}

1. Bray F, Ferlay J, Soerjomataram I, et al. Global cancer statistics 2018: GLOBOCAN estimates of incidence and mortality worldwide for 36 cancers in 185 countries. CA Cancer J Clin. 2018;68(6):394-424. doi: https://doi.org 10.3322/caac.21492. Erratum in: CA Cancer J Clin. 2020 Jul;70(4):313.

2. Instituto Nacional de Câncer José Alencar Gomes da Silva [Internet]. Rio de Janeiro: INCA; [data desconhecida]. Tipos de câncer: câncer do colo do útero; [modificado 2021 ago 24; acesso 2020 jan 10]. Disponível em: https:/www.inca.gov.br/tipos-de-cancer/cancer-docolo-do-utero

3. Carvalho PG, O’Dwer G, Rodrigues NCP. Trajetórias assistenciais de mulheres entre diagnóstico e início 
de tratamento do câncer de colo uterino. Saúde Debate. 2018;42(118):687-701. doi: https://doi. org/10.1590/0103-1104201811812

4. McGraw SL, Ferrante JM. Update on prevention and screening of cervical cancer. World J Clin Oncol. 2014;5(4):744-52. doi: https://doi.org/10.5306/wjco. v5.i4.744

5. Instituto Nacional de Câncer José Alencar Gomes da Silva. Estimativa 2020: incidência de câncer no Brasil [Internet]. Rio de Janeiro: INCA; 2019 [acesso 2020 jan 10]. Disponível em: https:/www.inca.gov.br/sites/ufu. sti.inca.local/files//media/document//estimativa-2020incidencia-de-cancer-no-brasil.pdf

6. Pereira AD. Percepção de mulheres frente ao diagnóstico e tratamento do câncer de colo uterino [dissertação na Internet]. São Luís, MA: Universidade Federal do Maranhão; 2019 [acesso 2020 jan 22]. Disponível em: https://tedebc.ufma.br/jspui/bitstream/tede/2703/2/ AndreaPereira.pdf

7. World Health Organization [Internet]. Geneva (Switzerland): WHO; [date unknown]. Breast cancer: prevention and control; c2009 [cited 2020 Jan 10]. Available from: https:/www.who.int/cancer/detection/ breastcancer/en/

8. Instituto Nacional de Câncer José Alencar Gomes da Silva [Internet]. Rio de Janeiro: INCA; [data desconhecida]. Controle do câncer de colo de útero: tratamento; [modificado 2021 jun 25; acesso 2020 jan 10]. Disponível em: https://www. inca.gov.br/controle-do-cancer-do-colo-do-utero/ acoes-de-controle/ tratamento\#: $\sim$ : text=Entre $\% 20$ os $\% 20$ tratamentos $\% 20$ mais $\% 20$ comuns, de $\% 20$ preserva $\%$ C3\%A7\%C3\%A3o\%20da\%20

9. Cibula D, Pötter R, Planchamp F, et al. The European Society of Gynaecological Oncology/European Society for Radiotherapy and Oncology/European Society of Pathology Guidelines for the management of patients with cervical cancer. Int J Gynecol Cancer. 2018 May;28(4):641-55. doi: https://doi.org/10.1097/ IGC.0000000000001216

10. Alves NG, Paz RMM, Arruda Junior OL, et al. Complicaçóes no tratamento de urolitíase devido a efeitos adversos da radioterapia pélvica. EJCH. 2020;12(9):e3594. doi: https://doi.org/10.25248/reas. e3594.2020

11. Amaral JD, Jesus ARBS. Uso terapêutico adjuvante de probióticos no efeito tardio da radioterapia pélvica: a retocolite ulcerativa. Braspen J [Internet]. 2018 [acesso 2020 jan 22];33(1):101-6. Disponível em: http:// arquivos.braspen.org/journal/jan-fev-mar-2018/16-ARUso-terapeutico-adjuvante.pdf

12. World Health Organization [Internet]. Geneva: WHO; c2021. WHOQOL: Measuring Quality of Life; [c2012]. [cited 2020 Jan 10]. Available from: https://www.who. int/toolkits/whoqol\#: - :text=WHO\%20defines $\% 20$
Quality $\% 20$ of $\% 20$ Life, $\% 2 C \% 20$ expectations $\% 2 C \% 20$ standards\%20and $\% 20$ concerns

13. Kyei KA, Yakanu F, Donkor A, et al. Quality of life among cervical cancer patients undergoing radiotherapy. Pan Afr Med J. 2020;35:125. doi: https://doi.org/10.11604/ pamj.2020.35.125.18245

14. Singh U, Verma ML, Rahman Z, et al. Factors affecting quality of life of cervical cancer patients: a multivariate analysis. J Cancer Res Ther. 2019;15(6):1338-44. doi: https://doi.org/10.4103/jcrt.JCRT_1028_17

15. Zhou W, Yang X, Dai Y, et al. Survey of cervical cancer survivors regarding quality of life and sexual function. J Cancer Res Ther. 2016;12(2):938-44. doi: https://doi. org/10.4103/0973-1482.175427

16. Correia RA, Bonfim CV, Ferreira DKS, et al. Quality of life after treatment for cervical cancer. Esc Anna Nery. 2018;22(4):e20180130. doi: https://doi. org/10.1590/2177-9465-ean-2018-0130

17. Yang L, Yuan J, Zeng X, et al. The outcomes and quality of life of young patients undergoing adjuvant radiotherapy versus non-radiotherapy following surgery treating early FIGO stage cervical squamous cell cancer in southwestern China. Sci Rep. 2020;10(1):9583. doi: https://doi.org/10.1038/s41598-020-66661-y

18. Sabulei C, Maree JE. An exploration into the quality of life of women treated for cervical cancer. Curationis. 2019;42(1):e1-e9. doi: https://doi.org/10.4102/ curationis.v42i1.1982

19. Mohanty SK, Chopra S, Mudaliar A, et al. A comparative analysis of quality of life after postoperative intensitymodulated radiotherapy or three-dimensional conformal radiotherapy for cervical cancer. Indian J Cancer. 2018;55(4):327-335. doi: https://doi.org/10.4103/ijc. IJC_453_17

20. Li LR, Lin MG, Liang J, et al. Effects of intrinsic and extrinsic factors on the level of hope and psychological health status of patients with cervical cancer during radiotherapy. Med Sci Monit. 2017;23:3508-17. doi: https://doi.org/10.12659/msm.901430

21. Kwak YK, Lee SW, Kay CS, et al. Intensity-modulated radiotherapy reduces gastrointestinal toxicity in pelvic radiation therapy with moderate dose. PLoS One. 2017;12(8):e0183339. doi: https://doi.org/10.1371/ journal.pone.0183339

22. Akbaba S, Oelmann-Avendano JT, Bostel T, et al. Percutaneous parametrial dose escalation in women with advanced cervical cancer: feasibility and efficacy in relation to long-term quality of life. Radiol Oncol. 2018;52(3):320-8. doi: https://doi.org/10.2478/raon2018-0029

23. Klopp AH, Yeung AR, Deshmukh S, et al. Patientreported toxicity during pelvic intensity-modulated radiation therapy: NRG oncology-RTOG 1203 . J Clin Oncol. 2018;36(24):2538-44. doi: https://doi. 
org/10.1200/JCO.2017.77.4273. Erratum in: J Clin Oncol. 2020 Apr 1;38(10):1118. doi: https://doi. org/10.1200/JCO.20.00346

24. Silveira CF, Regino PA, Soares MBO, et al. Qualidade de vida e toxicidade por radiação em pacientes com câncer ginecológico e mama. Esc Anna Nery. 2016;20(4):e20160089. doi: https://doi. org/10.5935/1414-8145.20160089

25. Correia RA, Bonfim CV, Feitosa KMA, et al. Disfunção sexual após tratamento para o câncer do colo do útero. Rev Esc Enferm USP. 2020;54:e03636. doi: https://doi. org/10.1590/s1980-220x2019029903636

26. Castaneda L, Bergmann A, Castro S, et al. Prevalência de incapacidades e aspectos associados em mulheres com câncer de colo do útero, Rio de Janeiro, Brasil. Cad Saúde Colet. 2019;27(3):307-15. doi: https://doi. org/10.1590/1414-462x201900030440

27. Cerentini TM, Schlöttgen J, Viana da Rosa P, et al. Clinical and psychological outcomes of the use of vaginal dilators after gynaecological brachytherapy: a randomized clinical trial. Adv Ther. 2019;36(8):1936-49. doi: https:// doi.org/10.1007/s12325-019-01006-4

28. Shankar A, Patil J, Luther A, et al. Sexual dysfunction in carcinoma cervix: assessment in post treated cases by LENTSOMA scale. Asian Pac J Cancer Prev. 2020;21(2):349-54. doi: https://doi.org/10.31557/ APJCP.2020.21.2.349

29. Yavas G, Yavas C, Dogan NU, et al. Pelvic radiotherapy does not deteriorate the quality of life of women with gynecologic cancers in long-term follow-up: a 2 years prospective single-center study. J Cancer Res Ther. 2017;13(3):524-32. doi: https://doi.org/10.4103/09731482.187243 